\title{
PROSTATECTOMÍA RADICAL ROBÓTICA: TÉCNICA DE LA UNIVERSIDAD DE CORNELL.
}

Juan I. Martínez-Salamanca, Sandhya Rao, Rajan Ramanathan y Ash Tewari.

Robotic Prostatectomy Program \& Urology Oncology Outcomes. Weill Medical College at Cornell University New York Presbyterian Hospital. Brady Urological Institute. New York. USA.

Resumen.- Presentamos en este capítulo nuestra técnica de Prostatectomía Radical Atérmica desarrollada en la Universidad de Cornell y el Instituto de Urología de la Universidad de Innsbruck desde el año 2004. Hasta el momento actual más de 600 pacientes han sido intervenidos por nosotros con el diagnóstico de cáncer de próstata clínicamente localizado. Describimos los pasos técnicos más importantes haciendo especial hincapié en la disección de los haces neurovasculares evitando el uso de cualquier tipo de energia térmica para evitar dañar los nervios cavernosos. Asimismo, se presentan los resultados patológicos y funcionales /continencia urinaria y potencia) de nuestra cohorte de 215 pacientes intervenidos durante el ano 2005.

Palabras clave: Robótica. Prostatectomía radical. Cáncer de próstata. Laparoscopia.
Summary.- We present in this chapter our Athermal Radical Robotic Prostatectomy technique developed in the Cornell University and the Urology Institute, Innsbruck University from 2004. Up to date, we have performed surgery in more than 600 patients diagnosed with clinically localized cancer of prostate. We describe most important technical steps, emphasizing in NVB dissection avoiding using any thermical energy not to injury the cavernous nerves. Additionally we present the pathological and functional outcomes /urinary continence and potency) of our cohort of 215 patients that underwent surgery during year 2005.

Keywords: Robotics. Radical prostatectomy. Cancer of prostate. Laparoscopy.

\section{INTRODUCCIÓN}

Se estima que en 2007 , se diagnosticará cáncer de próstata a más de 218.890 hombres en Estados Unidos y 27.050 morirán de la enfermedad.

La prostatectomía radical es el "Gold Standard" de tratamiento del cáncer localizado de próstata, pero se asocia con una importante morbilidad postoperatoria (1). A pesar de que el abordaje laparoscópico es una opción aceptada hoy, tiene una difícil curva de aprendizaje (2-4). La asistencia robótica proporciona al urólogo una visualización tridimensional, magnificación, instrumentación articulada, y movimientos intuitivos con reducción de escala, así como una postura más ergonómica. 
A pesar de que la técnica de la prostatectomía robótica esta bien descrita y ha quedado demostrada su eficacia y seguridad a corto plazo (5), sigue existiendo controversia en algunos principios que son diferentes a la técnica abierta (uso de energía para realizar la hemostasia, pérdida de sensación táctil, etc.). Presentamos aquí la técnica de prostatectomía radical robótica atérmica $(A R T \AA$, "Athermal Robotic Technique") que tiene como objetivo dar respuesta a estas preocupaciones. La técnica combina el respeto a los principios anatómicos expuestos por Walsh (6) y algunos detalles de la Técnica VIP® (Vatikutti Institute Prostatectomy) desarrollada por Menon (7).

\section{Los aspectos más importantes y novedosos de la mis- ma son:}

- Evita el uso de cualquier tipo de energía en la disección de las estructuras anatómicas implicadas en el resultado funcional (haces neurovasculares, vesículas seminales y disección apical).

- Considera la preservación de las estructuras incluidas en el concepto de anatomía "Trizonal" (Proximal Neurovascular Plate (PNP), Predominant Neurovascular Bundle (PNB) y Accessory Neural Pathways (ANP) $(8,9)$ (Figura 1).

- Incorpora una maniobra original para la identificación del cuello vesical ("Bladder Pinch®") (10).

- Describe la técnica de puboperineoplastia, (ART® (Anatomic Restoration Technique"), que mejora, en nuestra experiencia, los resultados de continencia precoz $(11,12)$.

\section{Desarrollo de la Técnica e Implantación}

La técnica fue desarrollada a través de una estrecha colaboración entre el Instituto de Robótica (Cornell University, NYC) y el Instituto de Urología de la Universidad de Innsbruck (Austria). Los pasos a grandes rasgos fueron los siguientes (8):

1. Disección cadavérica en el Instituto de Anatomía en Innsbruck (Austria).

2. Disección cadavérica posteriores de lo localizaciones anatómicas más precisas (Cornell University, NYC).

3. El equipo de cirugía robótica de Cornell visita Innsbruck para realizar y estandarizar la Técnica ART® en 15 pacientes.

4. Implementación de la técnica en la práctica diaria en el New York Presbyterian Hospital (Cornell University, NYC). Hasta la fechas en más de 600 pacientes desde enero de 2005.

\section{Estudios Anatómicos:}

El objetivo fundamental de los estudios cadavéricos fue hallar un mapa neural asociado a referencias anatómicas endoscópicas, en relación a los distintos pasos de la prostatectomía radical robótica (PRR) para la preservación del tejido nervioso alrededor de la zona de disección. Encontramos que el tejido neurovascular podía ser agrupado en 3 amplias zonas, dando lugar al concepto de anatomía "Trizonal" (9). (Figura 1).

Las indicaciones de la prostatectomía robótica son las mismas que las de la prostatectomía radical retropúbica. Son candidatos para este procedimiento los pacientes con cáncer de prostata clínicamente localizado y una expectativa de vida mayor a diez años, informados apropiadamente y que den su consentimiento por escrito. Aunque no existen contraindicaciones absolutas, ciertas condiciones pueden suponer un reto para el cirujano, especialmente en la fase de aprendizaje. Estas incluyen pacientes obesos (IMC > $35 \mathrm{~kg} / \mathrm{m} 2)$, cirugías abdominales o pélvicas previas con probabilidad de adherencias, hernias previas, gran volumen prostático (>100grs), tratamiento hormonal neoadyuvante y radioterapia previa.

\section{Descripción de la Técnica}

La técnica esta descrita para su utilización con el modelo da Vinci Surgical System de 3 brazos.

\section{Instrumentos utilizados}

Material robótico:

- 2 portagujas

- 1 pinzas bipolares tipo Maryland $\AA$

- 1 tijeras monopolares curvas (Hot Shears $®$ )

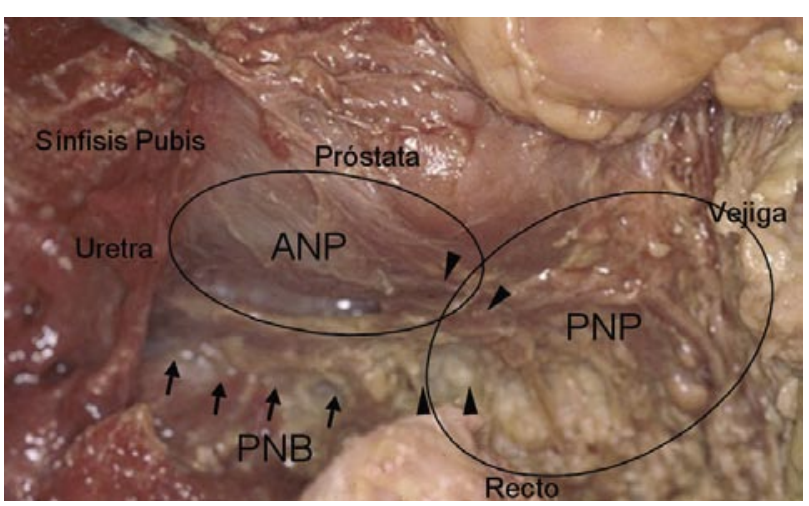

FIGURA 1. Concepto Anatomía Trizonal; ANP: Accesory Neural Pathways, PNP:Proximal Neurovascular Plate and PNP:Predominant Neurovascular Bundle. 
Material laparoscópico convencional inventariable:

- 1 portagujas

- 1 tijeras endoscópicas

- 2 pinzas de agarre tipo Grasper ${ }^{\circledR}$

- 1 aspirador/irrigador

- 1 dispositivo para extracción de la pieza tipo Endopouch $\circledast$

- Clips metálicos (5 y 10mm)

- Clips tipo Hemo-o-Lock® (5 y 10mm)

\section{Preparación preoperatoria y anestésica}

Aunque este apartado esta incluido y ampliamente desarrollado en la obra por el Dr. Mandhani en el Capítulo "Papel del ayudante quirúrgico en cirugía robótica" daremos aquí una pinceladas.

La intervención se realiza bajo anestesia general con intubación endotraqueal, generalmente con gases halogenados, más que con óxido nitroso, debido a que éste último puede producir distensión abdominal. Se recomienda, asimismo, la restricción de la administración intravenosa de líquidos a 600-800 $\mathrm{ml}$ hasta que se realiza la anastomosis vésico-uretral. Esta recomendación evita la producción excesiva de orina durante la cirugía, lo que reduce las maniobras de aspiración necesarias para mantener limpio el campo quirúrgico. La profilaxis antibiótica se realizará según cada protocolo hospitalario. Se recomienda asimismo la utilización de profilaxis tromboembólica peri operatoria y medias de compresión neumáticas en los miembros inferiores durante la cirugía.

El paciente se coloca en decúbito supino con ambos brazos pegados al cuerpo, con objeto de evitar el riesgo de lesiones del plexo braquial. Es recomendable un almohadillado torácico de gomaespuma sujeto con esparadrapo. Las extremidades inferiores se sitúan en posición de litotomía intermedia con las piernas separadas en flexión y abducción, y con un almohadillado bajo las nalgas. La separación de las piernas facilita la colocación del carro quirúrgico entre las mismas. Es imprescindible el almohadillado adecuado de todos los puntos de presión. La mesa de quirófano se coloca en posición de Trendelenburg extrema y a la menor altura posible. Finalmente se introduce una sonda Foley en la vejiga, en campo estéril.

\section{Acceso y creación del espacio de Retzius}

\section{Colocación de los puertos de acceso}

Creamos un neumoperitoneo mediante técnica de Veress, introducida a través del ombligo. Una vez insuflado, se amplia la incisión para colocar un trocar de $12 \mathrm{~mm}$., para la cámara. Se utiliza una lente $30^{\circ}$ para la exploración inicial de la cavidad y para visualizar la pared abdominal anterior durante la colocación de los puertos secundarios. Se colocan los puertos de $8 \mathrm{~mm}$. (instrumentos robóticos), uno a cada lado a $8-10 \mathrm{~cm}$., de la línea media y alrededor de $2.5 \mathrm{~cm}$., por debajo del ombligo. Se introduce un puerto de $5 \mathrm{~mm}$., entre el puerto de la cámara y el puerto de $8 \mathrm{~mm}$., del lado derecho. Se coloca un puerto de $12 \mathrm{~mm}$., en el lado derecho de la línea axilar media, $2.5 \mathrm{~cm}$. por encima de la cresta ilíaca. Se coloca otro puerto de $5 \mathrm{~mm}$., simétrico y contralateramente al puerto de $12 \mathrm{~mm}$. (Figura 2).

\section{Desarrollo del Espacio de Retzius}

Colocamos la cámara, a través del puerto de $12 \mathrm{~mm}$. Si es necesario se realiza adhesiolisis previa. Se movilizan el colon sigmoide y el ciego, mediante incisión del peritoneo parietal. Se identifican el ligamento umbilical medio, uraco, conductos deferentes y el anillo inguinal interno y se utilizan como puntos de referencia para una disección posterior. Se puede observar el conducto deferente transcurriendo lateralmente al anillo inguinal interno y se realiza una incisión vertical sobre él, medial al anillo inguinal interno y lateral a los ligamentos medios umbilicales. Se secciona el conducto deferente. Profundizando la incisión, se separa la grasa y el tejido laxo, cauterizando los pequeños vasos que puedan aparecer hasta exponer la fascia trasversalis. Se identifica la vena ilíaca externa. La disección se debe realizar medial a los vasos epigástricos.

Se realiza una disección idéntica en el lado opuesto. Las dos incisiones verticales se unen por encima de la línea media en forma de " $U$ " invertida por encima del pubis. Se profundiza la incisión, dejando la sínfisis púbica completamente expuesta. Mientras se desciende la vejiga, se debe cuidar de no dañar la fascia trasversalis posterior y se limpia la cara anterior prostática y la fascia endopélvica, retirando y coagulando la grasa existente. El cumplimiento de estos principios es importante para la creación de un

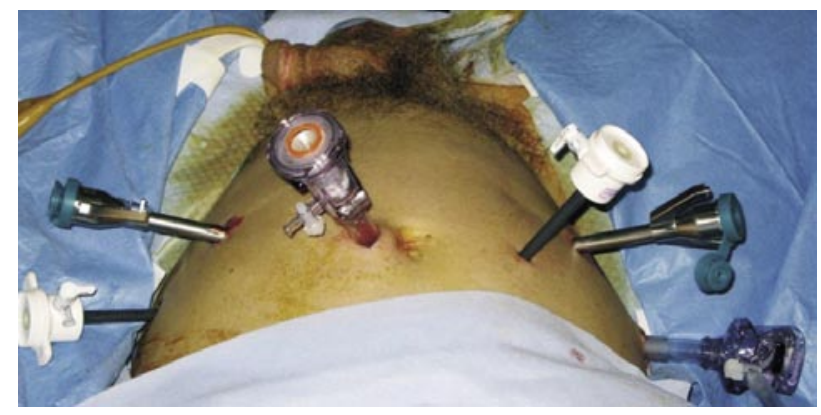

FIGURA 2. Colocación de los puertos de entrada. 
campo operatorio adecuado. Esto es particularmente crítico en circunstancias difíciles como obesidad, pelvis estrecha, próstata grande, cirugía pélvica 0 inguinal previa, reintervención, o cualquier combinación de todas ellas.

\section{Exposición del Ápex y la Fascia Endopélvica}

Una vez que la sínfisis púbica está expuesta, se retira la grasa para poder ver las fibras brillantes de la fascia endopélvica. Asimismo, se retira la grasa cerca del ápex de la próstata para mostrar los ligamentos puboprostáticos y el complejo venoso dorsal (CVD). No abordamos el CVD ahora, lo hacemos al final de la intervención justo antes de la sección uretral cuando la próstata sea móvil.

La fascia endopélvica se abre usando el extremo afilado de las tijeras robóticas multi-articuladas. Se utiliza un único gesto para abrir la fascia y evitar la cauterización (Movimiento "Samurai") (Figura 3). Se realiza una incisión distal mínima de la fascia mediante corte medial del arco tendíneo y recorriendo la fascia del elevador por fuera de la superficie lateral de la próstata. La incisión se limita a aproximadamente un tercio proximal de la próstata, siendo el límite distal la extensión con forma de hoz de los ligamentos puboprostáticos. Se preservan estos, evitando la disección lateral. Se ven entonces, las fibras del elevador del ano. En este momento, las adherencias laterales del ápex se encuentran parcialmente liberadas. La imagen tridimensional y la magnificación proporcionadas por el sistema robótico ayudan a controlar las hemorragias menores y a lograr un acceso seguro a los espacios paraprostáticos. Al final de esta etapa, se ven los ligamentos puboprostáticos intactos en continuidad con la fascia en ambos lados.

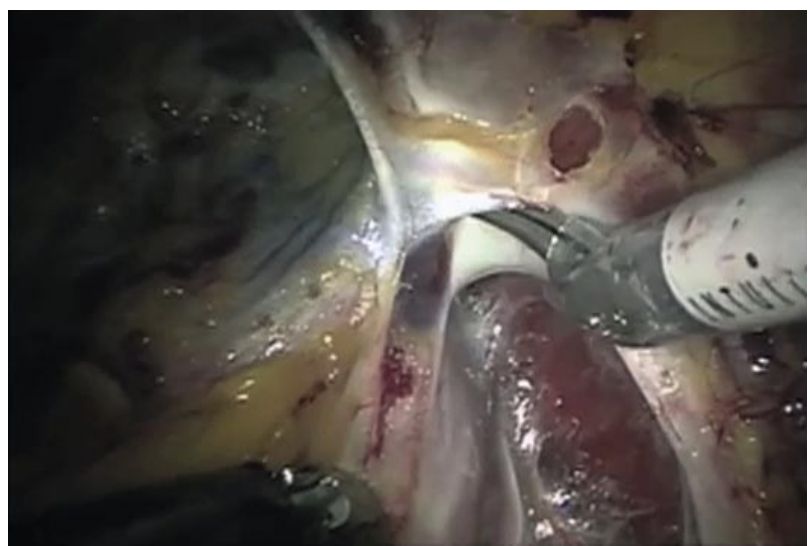

FIGURA 3. Apertura de la fascia endopélvica (Movimiento del "Samurai").

\section{Sección del Cuello Vesical ("Bimanual Bladder Neck Pinch") (10). \\ La sección del cuello anterior de la vejiga,} encontrando un cruce exacto entre la próstata y las fibras del detrusor y descubriendo las vesículas seminales en el plano posterior son los nuevos retos de los cirujanos que comienzan su andadura en la PRR. La disección se realiza de forma anterógrada, a diferencia de la técnica abierta que implica abordar el ápex antes de realizar la disección del cuello de la vejiga y las vesículas seminales. También es diferente al abordaje laparoscópico clásico que diseca las vesículas seminales a través del fondo de saco de Douglas antes de la sección del cuello vesical (13).

La sutil y gradual transición de la vejiga a la próstata hace difícil en muchas ocasiones, la identificación precisa de la unión próstato-vesical. Las fibras anteriores del detrusor se continúan distalmente para fusionarse con los ligamentos puboprostáticos y a menudo contienen ramas del complejo venoso dorsal. En la región lateral, existe una fusión de la vejiga, el pedículo prostático, la arteria vesical inferior, el PNP (Proximal Neurovascular Plate), la fascia del elevador y la lateroprostática. Posteriormente las fibras del cuello de la vejiga se continúan distalmente con la lámina retrotrigonal y se fusionan con la parte proximal de la próstata y el estuche seminal.

En la cara anterior (unión prostato-vesical) se encuentran fibras del llamado "delantal del detrusor" mezclado con venas provenientes del CVD e inmediatamente por debajo, la cara anterior del cuello vesical (unión la mucosa del cuello con la uretra prostática). El paso siguiente será localizar el cuello vesical y abrirlo controlando la posible hemorragia proveniente de las extensiones venosas del CVD. Para facilitar

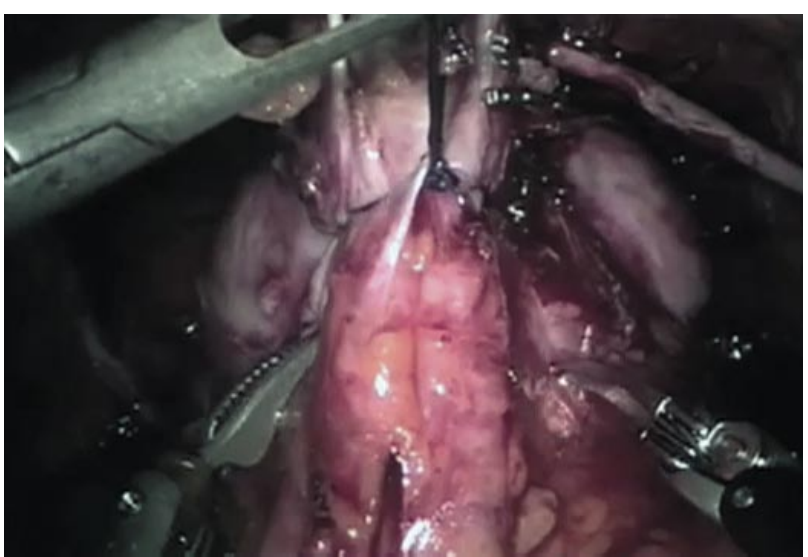

FIGURA 4. Maniobra de identificacion del cuello vesical ("Bimanual Bladder Neck Pinch"). 
este paso hemos desarrollado una técnica conocida como "Bimanual Bladder Neck Pinch"[10], que pasamos a describir a continuación. (Figura 4).

Esta parte de la disección se realiza con una lente de $30^{\mathrm{a}}$ orientada inferiormente. Colocamos un punto de sutura de Vicry|®/O en la superficie anterior de la próstata, proximal a los ligamentos puboprostáticos para prevenir la hemorragia venosa retrógrada y también para traccionar. Otro punto de sutura (proximal al anterior) se colocará sobre la cara anterior de la vejiga, que también servirá de tracción. Usando las pinzas robóticas tipo Maryland y tijeras robóticas se sujeta la próstata por los dos lados y se mueven las manos proximalmente y medialmente como resbalando de delante hacia detrás. Asímismo al mismo tiempo el ayudante tracciona de la sonda vesical anclando el globo en el cuello. En este punto la unión prostatovesical se puede identificar fácilmente. El asistente situado en el lado izquierdo tira de la sutura de tracción prostática hacia los pies del paciente. El cirujano proporciona una contratracción firme con las pinzas tipo Maryland. Entonces se realiza una incisión en el cuello anterior de la vejiga, con una forma curvilínea. Se profundiza la disección hasta que se ve la sonda vesical. (Figura 5).

La identificación de la sonda asegura que la incisión del cuello anterior de la vejiga se ha hecho de forma apropiada. Se retira el catéter de la vejiga. El balón se desinfla y el asistente del lado izquierdo sujeta la punta de la sonda y tracciona firmemente de ella. La incisión anterior se amplia lateralmente con coagulación en el plano fibrograso entre la vejiga y la próstata. Mediante tracción axial de la sonda, se podrá ver la localización exacta de la incisión poste-

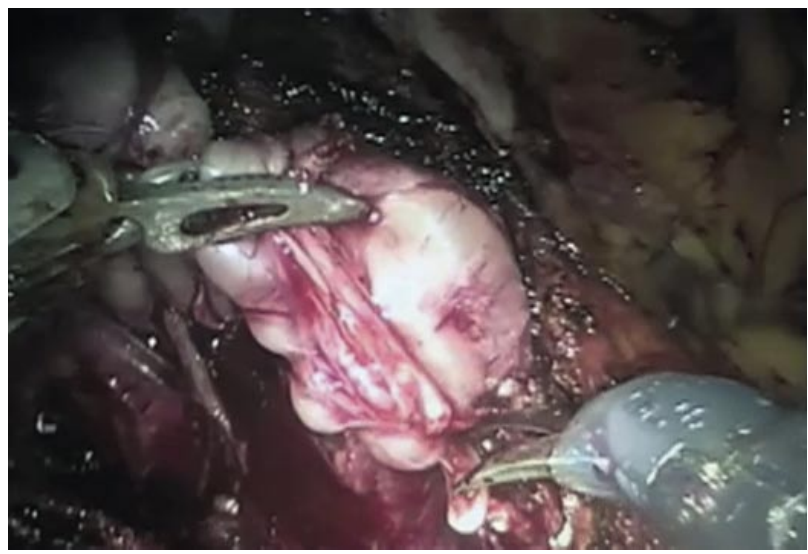

FIGURA 5. Apertura y disección del cuello vesical. rior y así se podrá realizar una incisión precisa de la mucosa posterior del cuello vesical.

Se deben tomar todas las precauciones posibles para preservar la anatomía del cuello vesical y evitar dañar los orificios ureterales. La incisión posterior se modifica de acuerdo con el tamaño y la morfología de la próstata. Se profundiza la incisión manteniéndola tangencial a la próstata y evitando cualquier incisión del parénquima prostático. La disección vertical protege contra una posible lesión del trígono o creación de un ojal en la vejiga. Si durante este paso, se observa mucha hemorragia puede ser signo de que se está entrando en la próstata. Se retira el catéter de la uretra y el asistente del lado izquierdo retrae anteriormente la base prostática posterior. Se desarrolla entonces un plano detrás de la pared posterior del cuello de la vejiga, que expone la capa retrotrigonal (14). Cortando esta capa se abre una ventana a través de la cual se ven los conductos deferentes y las vesículas seminales. A partir de este momento en adelante, se evita la electro cauterización.

\section{Disección de las vesículas seminales y de los con- ductos deferentes}

En continuidad con los conductos deferentes y las vesículas seminales se encuentra una lámina fibromuscular firme, posterior al cuello de la vejiga y anterior a la fascia de Denonvillier. Se amplia la incisión lateralmente para permitir la retracción del cuello de la vejiga cranealmente. El asistente del lado izquierdo levanta la base de la próstata, utilizando pinzas de agarre (Endograsper®). Los pequeños vasos deferenciales se controlan mediante clips quirúrgicos, evitando el uso de cualquier tipo de energía.

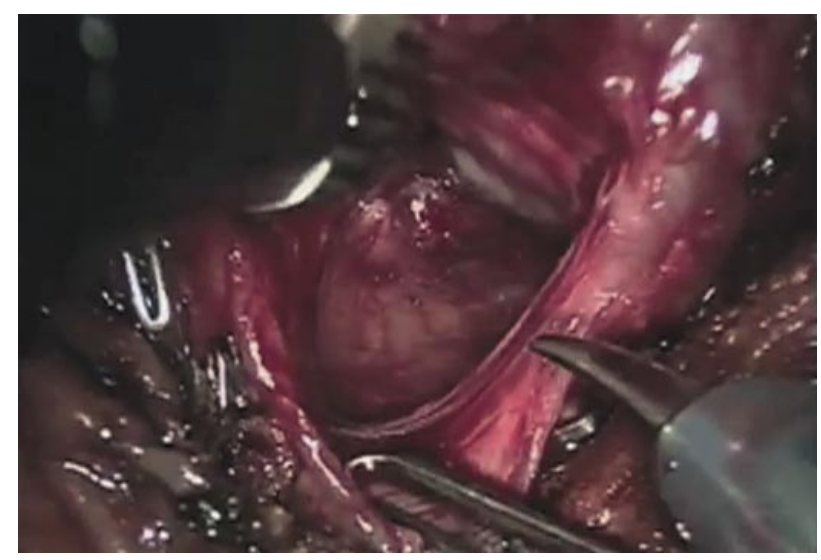

FIGURA 6. Disección de los conductos deferentes y vesículas seminales. Uso de clips (metálicos y Hemo-o-Lock $\circledast$ ). Técnica Atérmica. 
Los conductos deferentes se clipan y dividen mediante aplicación de Hemo-o-Lock®. (Figura 6).

La disección de las vesículas seminales comienza desde el plano avascular medial. El PNP se localiza a $5-10 \mathrm{~mm}$. (promedio $5 \mathrm{~mm}$.) lateralmente a las vesículas seminales y existe el peligro de lesión si se aplica cualquier tipo de energía o se incluyen dentro del tejido controlado por alguno de los clips. Por tanto, esta parte de la disección se realiza de forma atérmica, desarrollando pequeños pedículos y controlándolos con clips quirúrgicos de $5 \mathrm{~mm}$., o suturándolos cerca de la superficie de la vesícula seminal. Ambas vesículas seminales y conductos deferentes son traccionados medialmente. Esto permite exponer la superficie posterior de la próstata.

La clave para una exitosa preservación nerviosa es una disección meticulosa, permaneciendo cerca de la vesícula seminal y evitando disecar las capas exteriores, mantener una buena visión, realizar un adecuado control de los vasos y evitar el uso de la coagulación.

\section{Incisión de la fascia de Denonvillier y posterior di- sección hasta el ápex}

El tejido neurovascular forma dos arcos laterales con el espacio pre-rectal en el centro. Mediante tracción de ambos conductos deferentes y vesículas seminales, se realiza una incisión en forma de " $U$ " invertida en la fascia de Denonvillier, continuándola bajo la superficie prostática. Se deja una hoja central pegada a la próstata, mientras que lateralmente se deja otra hoja alrededor del haz neurovascular. La incisión se realiza de manera precisa y controlando los vasos en el espacio pre-rectal. (Figura 7).

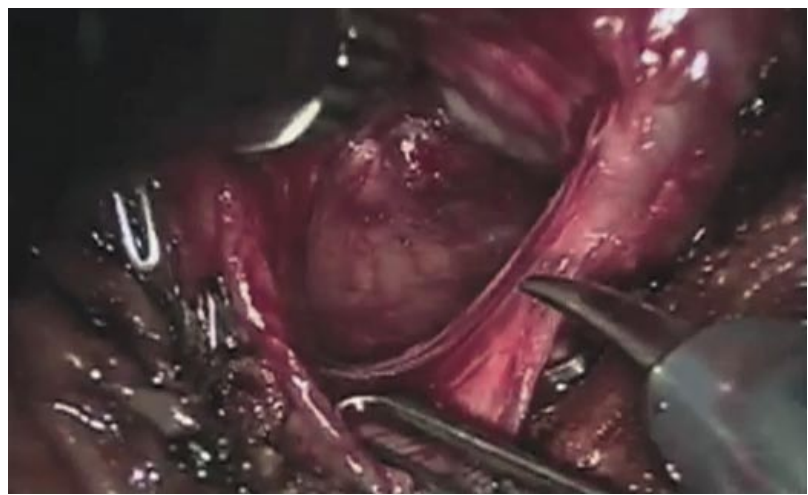

FIGURA 7. Creación del plano posterior prerrectal. Apertura de la fascia de Denonvillier.
Se debe tener cuidado de dejar las dos capas de la fascia de Denonvillier en el espécimen y descubrir la grasa pre-rectal. Se continúa la disección distalmente hasta el ápex y lateralmente hasta los haces para descubrir el triangulo neurovascular. (Figura 8).

\section{Control de ambos pedículos laterales}

Se ejerce una tracción superior de los conductos deferentes y vesículas seminales así como de la próstata. Esto provoca que los pedículos prostáticos se observen más claramente a ambos lados. Diferenciar el pedículo prostático del PNP y del PNB supone, a menudo, un reto para el cirujano. Algunas veces el plano se diseca fácilmente entre el pedículo prostático y el haz neurovascular. Otras veces debemos crear un plano con las pinzas y después ir colocando clips para controlar todos los vasos. La clave es permanecer cerca de la próstata, disecar los vasos en pequeños paquetes, identificarlos entrando en la próstata y controlarlos atérmicamente. (Figura 9).

Es preferible utilizar pinzas pequeñas y control de los pedículos individualmente para evitar la electro cauterización y la ligadura en bloque. La orientación, tamaño, y extensión del pedículo varía significativamente dependiendo de la anatomía de la próstata y la neovascularización producida por el tumor.

\section{Liberación de los haces neurovasculares (Figuras} $10,11,12$ y 13$)$.

Con la próstata traccionada lateralmente se expone la fascia pélvica lateral. Las capas de la fascia peri prostática se fusionan con la capa anterior de la fascia de Denonvillier lateralmente a la próstata para formar un potencial espacio triangular conteniendo los haces neurovasculares. La capa interna

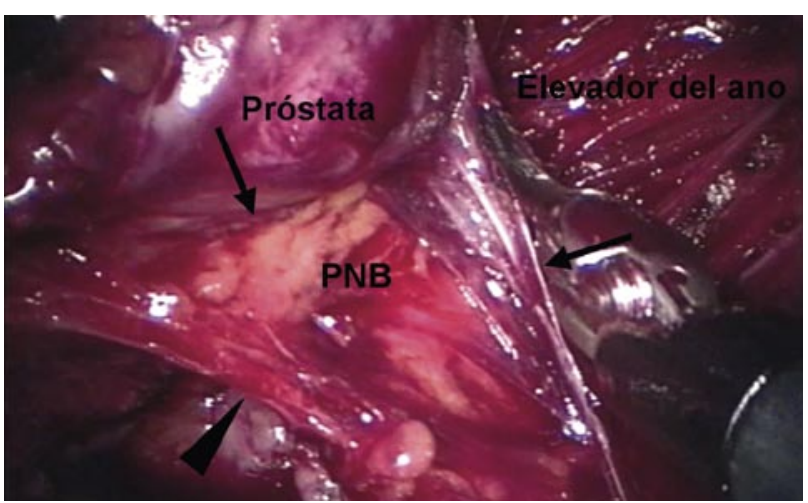

FIGURA 8. Triángulo neurovascular; PNP: "Predominant Neurovascular Bundle". 


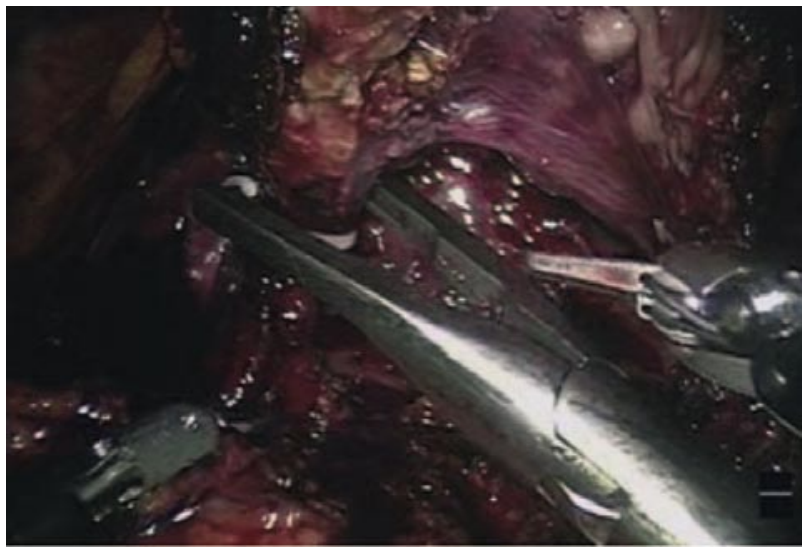

FIGURA 9. Control mediante clips tipo Hem-o-Lock® del pedículo prostático izquierdo.

de la fascia peri prostática forma la pared vertical medial de este triángulo; (Figura 8) la capa externa - lateral de la fascia pélvica forma la pared lateral y la capa anterior de la fascia de Denonvillier forma la pared posterior. Entrando en este espacio triangular entre la fascia de Denonvillier posteriormente, fascia pélvica lateral, y la próstata medialmente se puede preservar el haz neurovascular (abordaje interfascial). El cirujano tiene que dejar disecada la fascia pélvica lateral fuera de la próstata. Se hace la incisión en un plano superficial a la fascia prostática desde el ápex a la unión próstata-vesical, permaneciendo siempre paralelo a los haces neurovasculares. Con suaves movimientos, se liberan de manera roma los haces de la próstata. Esta maniobra libera los haces y proporciona puntos de referencia para una posterior disección anterógrada. La disección es totalmente atérmica uti-

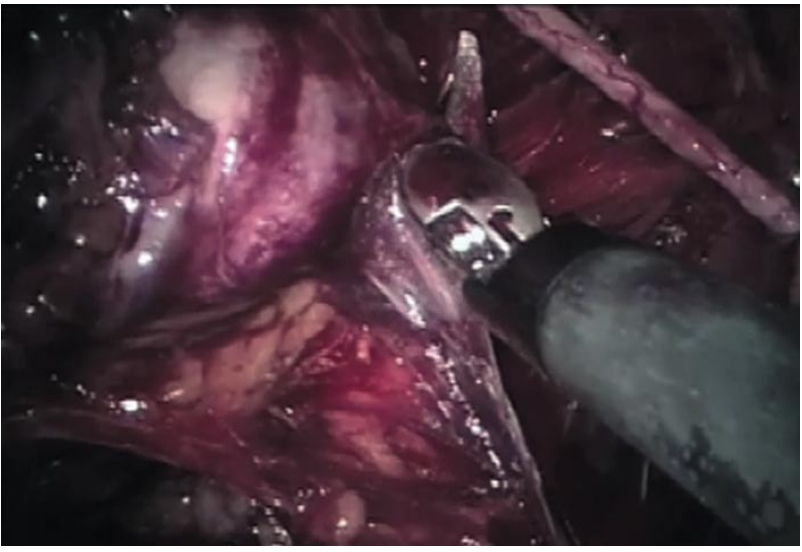

FIGURA 10. Disección de la fascia prostática lateral. Preservación neurovascular.

lizando clips (metálicos o tipo Hemo-o-Lock®) para cualquier control vascular. La disección se continúa distalmente hasta el ápex y lateralmente. La disección adicional continua dentro del triángulo para liberar los haces permaneciendo cerca de la fascia prostática. Una vez en el ápex, se controlan mediante clips algunos vasos perforantes al mismo, permitiendo liberarlo. En casos muy seleccionados, realizamos abordaje intrafascial, es decir el plano por debajo de la fascia prostática. (Figura 12).

\section{Disección Apical, Control del CVD y Sección Ure-} tral

En la disección del ápex se preservan los ligamentos puboprostáticos. La próstata se libera, mediante corte frío y disección roma sin utilizar la cauterización. La uretra y sus estructuras de sopor-

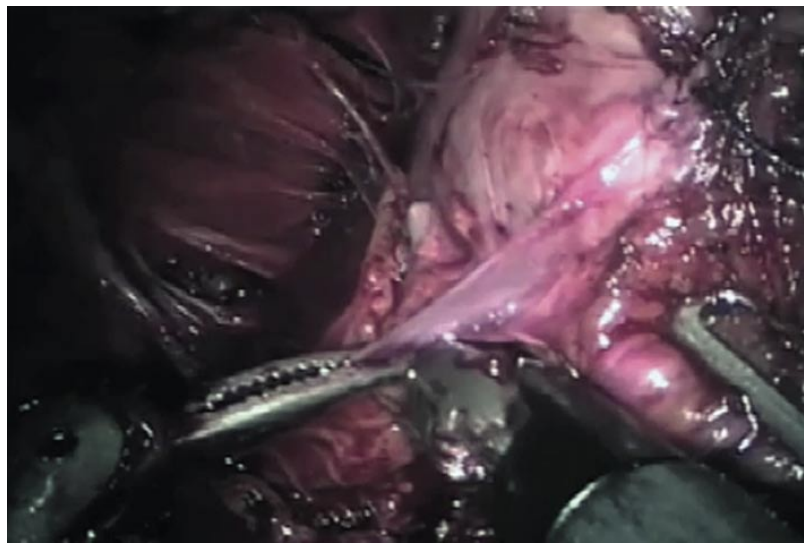

FIGURA 11. Ejemplo de disección extrafascial en el lado izquierdo.

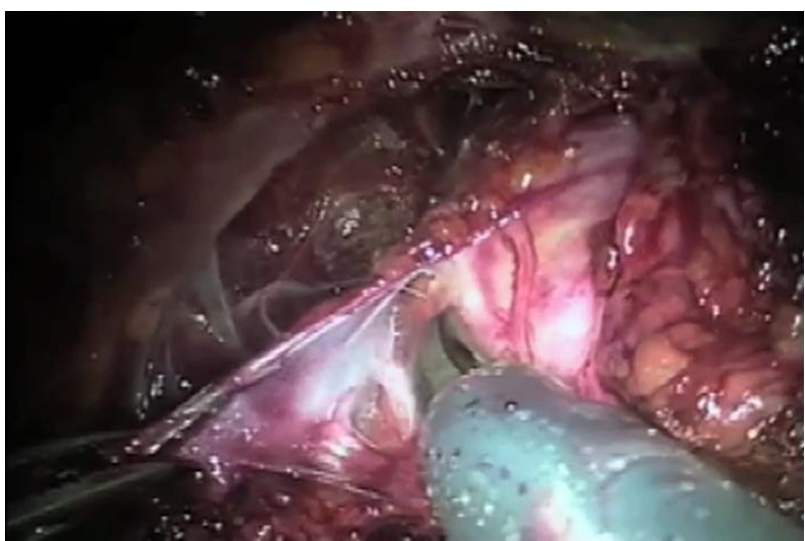

FIGURA 12. Ejemplo de disección intrafascial en lado derecho. 


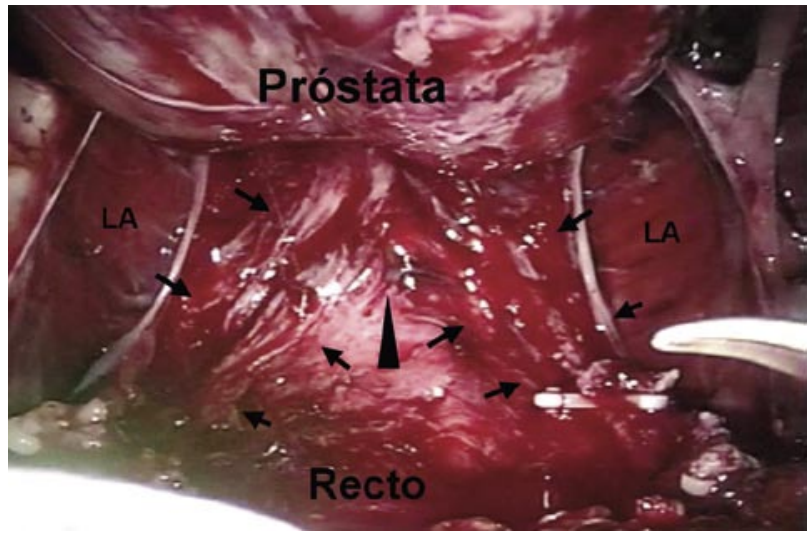

FIGURA 13. Disección roma del plano posterior.

te (lateralmente la fascia endopélvica, anteriormente los ligamentos puboprostáticos y el músculo puboperineal como hamaca posterior) quedan intactas. (Figura 14).

Se tracciona próximamente de la próstata y se controla el CVD. Mediante sutura de Vicryl® se pasa sobre la vena dorsal y por debajo de ligamentos puboprostáticos en la derecha y luego se saca por la izquierda por encima de la vena dorsal y por debajo del ligamento. (Figura 16). Se hacen dos de estas pasadas y luego se anuda. De esta manera el CVD se controla tomando todas las precauciones para evitar incluir los ligamentos puboprostáticos y siempre intentando alejarse del esfínter externo. La próstata se tracciona en dirección proximal y usando las tijeras de punta redonda, el CVD y la uretra anterior se cortan próximamente a los ligamentos puboprostáticos. La uretra posterior se corta asegurando la disección y separación de los haces neurovasculares. El objetivo final es que el muñón uretral sea tan largo y robusto como sea posible, sin comprometer la seguridad en el margen apical. (Figuras 14 y 16). El arco tendíneo, la hoja lateral de la fascia endopélvica y los ligamentos puboprostáticos preservados en ambos lados forman un collar de tejido alrededor del muñón uretral tras la extirpación de la glándula. Se toman biopsias intraoperatorias del margen uretral y se espera su resultado. Casi todo el tejido neurovascular converge en el ápex y el plexo distal y anterior puede dañarse durante la sección uretral y la anastomosis. Los ángulos visuales se cambian varias veces para permitir la identificación de ambos haces y su relación con el esfínter.

\section{Linfadenectomía pélvica bilateral}

La linfadenectomía pélvica se realiza con una lente de $0^{\circ}$. Realizamos linfadenectomía ílio-obtu- ratriz en todos los pacientes. Los límites son; distal, el ligamento de Cooper, proximal, la bifurcación ilíaca, inferior, el nervio obturador y superior, el borde de la vena ilíaca externa. Los instrumentos utilizados habitualmente son la pinza bipolar tipo Maryland bipolar y la tijera curva monopolar.

\section{Anastomosis Uretrovesical}

La anastomosis uretrovesical se realiza con sutura continua. Se utilizan dos suturas monofilamento (Monocrylß) de $22 \mathrm{~cm}$ (11 cm cada una) 3-0 en

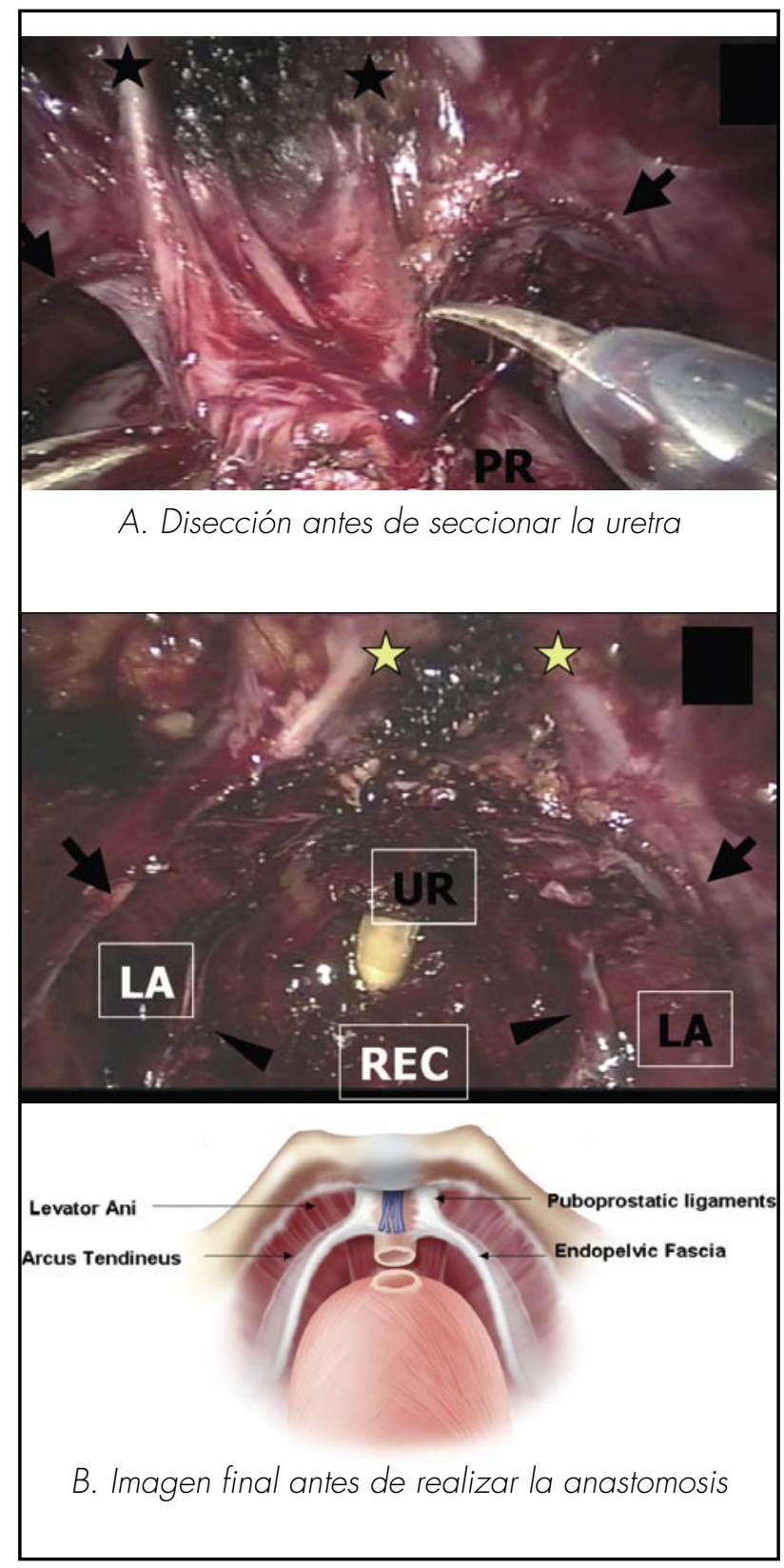

FIGURA 14. Disección apical y sección uretral. 


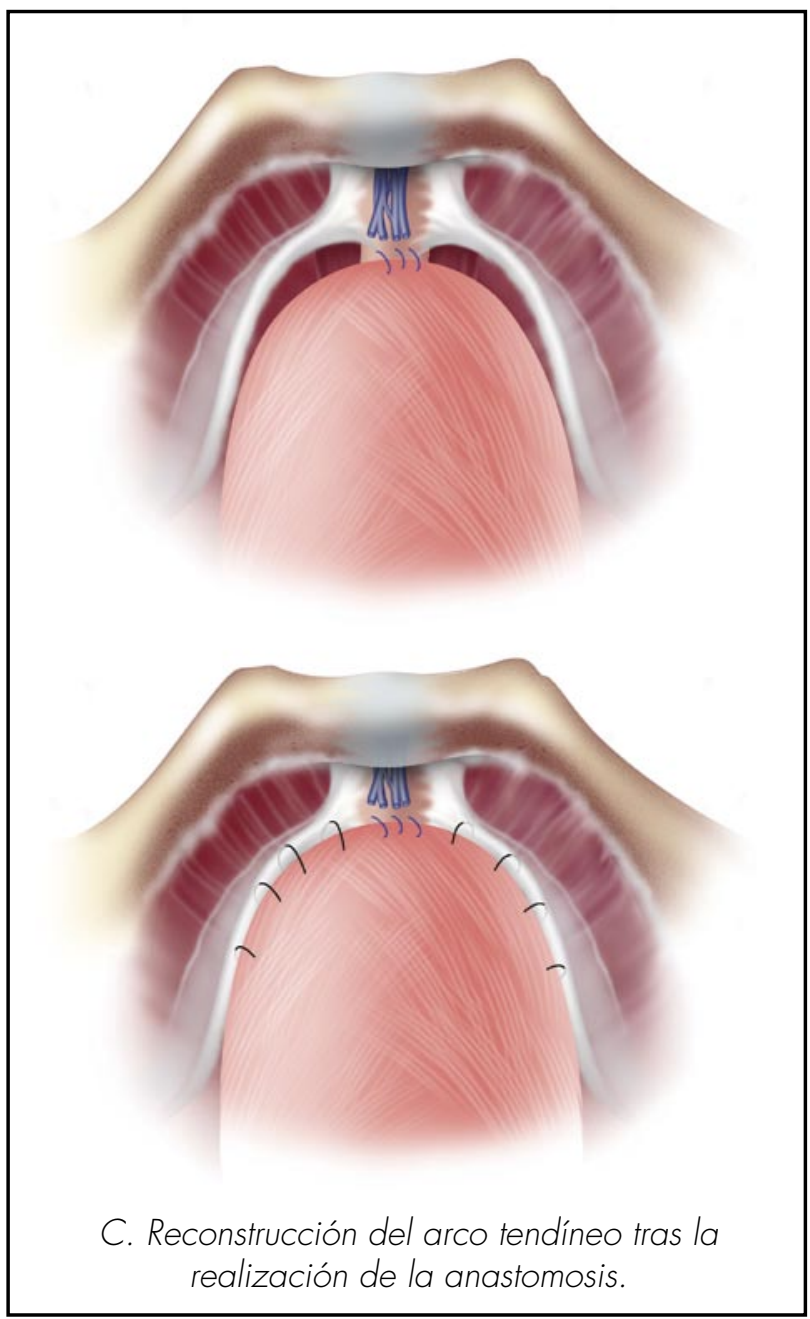

FIGURA 15. Técnica de Puboperineoplastia (PPP). encuentra con la sutura coloreada. Se cortan las agujas y se anudan juntos los dos extremos con varios nudos. Durante toda la anastomosis, el asistente de lado derecho juega un importante papel, moviendo la sonda dentro y fuera, según se requiera exponer o no la mucosa uretral.

Una vez completada la anastomosis realizamos la técnica de Puboperineoplastia (ART® "Anatomic Restoration Technique") $(11,12)$. (Figuras 14 y 15). La reconstrucción del arco tendíneo y la puboperineoplastia se realizan mediante sutura continua, empezando proximal al cuello de la vejiga. Se realizan 3 pasadas de hilo en cada lado entre el collar puboprostático que se ha respetado y la vejiga, cerca de la anastomosis. La sutura aproxima distalmente la vejiga y la anastomosis vesicouretral al arco tendíneo, ligamentos puboprostáticos, músculo puboperineal y tejido conectivo medial de cada lado.

El objetivo es restaurar tanto el soporte periuretral como el ángulo vésicouretral. Este paso lleva aproximadamente de 2 a 5 minutos y la sutura utilizada es Vicry|ß 2/0 con aguja RB 1. El tiempo medio de la anastomosis es de 12 minutos (rango 8-15 minutos). (Figura 17).

Se inserta un catéter 18F tipo Foley, y se comprueba la estanqueidad de la anastomosis con la introducción de $200 \mathrm{~mL}$ de solución salina. Se deja un drenaje $14 \mathrm{~F}$ Jackson-Pratt ${ }^{\circledR}$ para extraer cualquier colección que pueda haberse acumulado en el abdomen superior, debido a la posición de Trendelenburg.

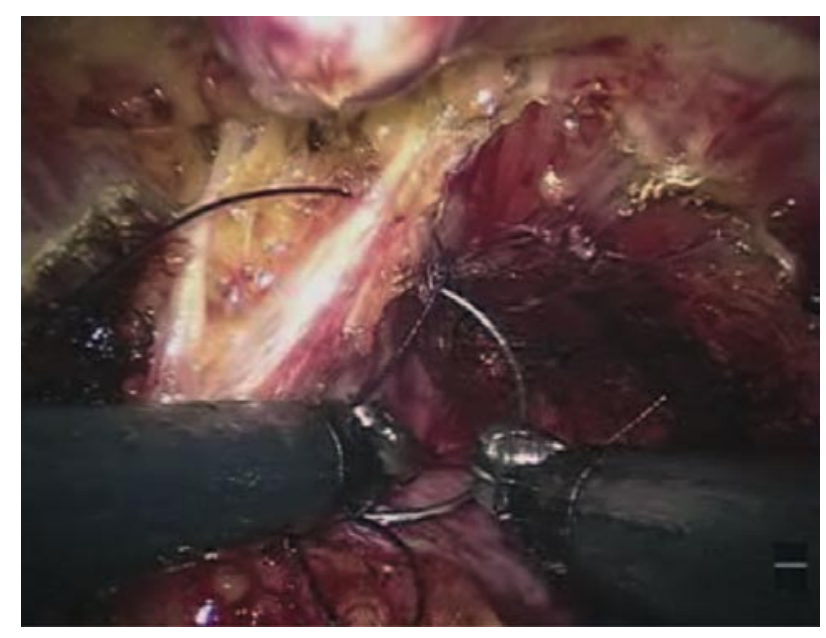

FIGURA 16. Ligadura complejo venoso dorsal. una aguja tipo RB, una de coloreada y otra transparente. Sus extremos se atan extracorpóreamente haciendo 10 nudos. La anastomosis se comienza por la sutura coloreada y se procede en sentido horario, asegurando primero la cara posterior. El primer punto se realiza a las 5 en el cuello posterior de la vejiga de fuera a dentro y se continúa en la uretra de dentro a afuera. (Figura 17). Después de los primeros tres puntos, se anuda la sutura con tres nudos, llevando la vejiga abajo hacia la uretra y formando la placa posterior. Se continúa la anastomosis siguiendo las agujas del reloj hasta la posición de las 11, donde se sujeta bajo tracción, bien por el asistente o bien insertando una aguja en la pared pélvica lateral.

Se completa ahora el resto de la anastomosis con sutura sin color en el sentido contrario a las agujas del reloj. Se pasa primero de fuera a dentro en la uretra y de dentro a fuera de la vejiga en la posición de las 4 hasta la posición de las 11 , donde se 


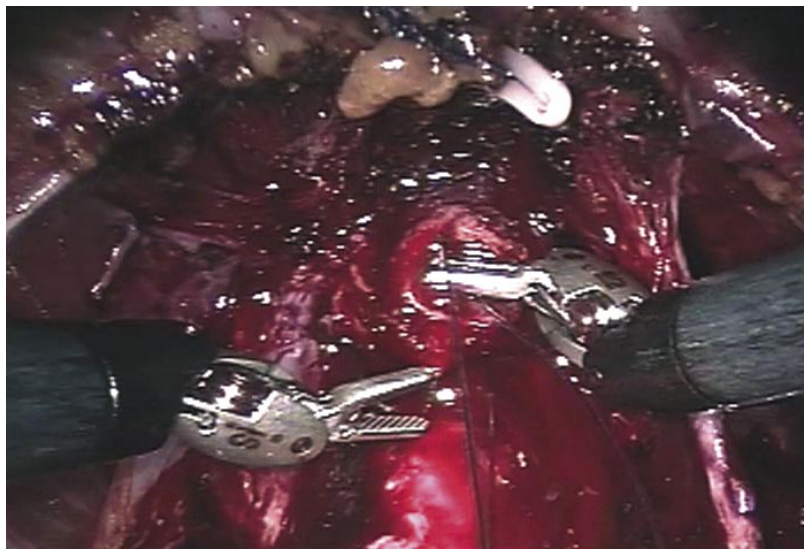

FIGURA 17. Aspecto del muñón uretral. Inicio de la anastomosis uretrovesical.

La técnica finaliza con el embolsamiento y extracción del espécimen (Endocatch $\circledast$ ) y cierre de los puertos.

\section{RESULTADOS}

\section{Resultados Oncológicos}

Los resultados oncológicos incluyen los pacientes intervenidos durante 2005, un total de 215. Los resultados se muestran en las Tablas I-V.

\section{Resultados Funcionales (Potencia Sexual y Continen- cia Urinaria)}

Entre enero de 2005 a mayo de 2006, analizamos nuestra experiencia en un total de 324 pacientes intervenidos de PRR. Los valores iniciales en cuanto a datos demográficos se recogieron prospectivamente e incluían edad, raza, índice de masa corporal (IMC), PSA, volumen de la próstata, escala de Gleason, porcentaje de cáncer en la biopsia, TNM estadio clínico, co-morbilidad, y escalas IPSS y IIEF.

Las variables postoperatorias estudiadas incluyeron cifra de hematocrito al alta, días hospitalización, estadio patológico, escala de Gleason, margenes quirúrgicos, duración de la cateterización, retención urinaria, complicaciones, estado de la continencia y de la función sexual. Todos los pacientes completaron voluntariamente cuestionarios confidenciales relacionados con la función eréctil (EPIC y IIEF) antes de la intervención y al mes, 3, 6, y 12 meses después de la cirugía. Se definió la potencia como la existencia de erecciones lo suficientemente firmes como para la penetración vaginal.
TABLA I. CARACTERISTICAS DE LOS PACIENTES.

\begin{tabular}{|c|c|c|}
\hline Mediana Edad \pm SD (rango) & \multicolumn{2}{|c|}{$60 \pm 6(45-75)$} \\
\hline No preop PSA (ng/ml) (\%) & & \\
\hline$\bullet<2,5$ & 19 & (8.9) \\
\hline - $2.6-4.0$ & 43 & $(20$. \\
\hline - $4.1-10.0$ & 135 & $(63.4)$ \\
\hline - $10.1-20.0$ & 12 & $(5.6)$ \\
\hline$\cdot>20$ & 4 & 11 \\
\hline
\end{tabular}

No estadio clínico (cTNM) (\%)

- Tla-b

- Tlc

- $\mathrm{T} 2 \mathrm{a}$

- $\mathrm{T} 2 \mathrm{~b}$

- $\mathrm{T} 2 \mathrm{c}$

- T3a

- T3c

1

No Gleason biopsia (\%)

- 6

154

- $3+4$

- $4+3$

- $\geq 8$

No + invasión perineural $(\%)$

16

No PIN alto grado(\%)

38

Mediana No cilindros \pm DS

$12 \pm 8(1-32)$ (rango)

Mediana No cilindros (cil)

$2 \pm 2(1-15)$

positivos \pm DS (rango)

- No 1 cil + (\%)

- Mediana

Gleason

6

- No 2 cil + (\%)

$58(27.0)$

- Mediana

Gleason

6

Mediana Max \% tumor \pm DS $\quad 15 \pm 25$ (1-95) (rango) 
TABLA II. DATOS PATOLÓGICOS (PIEZA QUIRÚRGICA)

\begin{tabular}{|c|c|c|}
\hline Mediana peso Próstata \pm DS & \multicolumn{2}{|c|}{$48 \pm 34(16$} \\
\hline (rango) & & $346)$ \\
\hline \multicolumn{3}{|l|}{ No estadio patológico (\%) } \\
\hline - $\mathrm{pT} 2 \mathrm{a}$ & 37 & $(17.3)$ \\
\hline - $\mathrm{pT} 2 \mathrm{~b}$ & 7 & (3.3) \\
\hline - $\mathrm{pT} 2 \mathrm{c}$ & 144 & (67.3) \\
\hline - $\mathrm{pT3a}$ & 16 & $(7.5)$ \\
\hline - pT3b & 8 & (3.7) \\
\hline - PT4 & 2 & (0.9) \\
\hline \multicolumn{3}{|l|}{ No Gleason pieza (\%) } \\
\hline$\bullet<6$ & 2 & $(1.0)$ \\
\hline - 6 & 98 & $(46.0)$ \\
\hline - $3+4$ & 88 & $(41.3)$ \\
\hline - $4+3$ & 13 & (6.1) \\
\hline$\bullet \geq 8$ & 12 & $(5.6)$ \\
\hline \multicolumn{3}{|l|}{ No estadio clínico (cTNM) (\%) } \\
\hline - Tla-b & 1 & $(0.5)$ \\
\hline$\bullet$ Tlc & 159 & $(74.6)$ \\
\hline - T2a & 29 & $(13.6)$ \\
\hline - $\mathrm{T} 2 \mathrm{~b}$ & 10 & (4.7) \\
\hline - $\mathrm{T} 2 \mathrm{c}$ & 12 & $(5.6)$ \\
\hline - T3a & 1 & (0.5) \\
\hline - T3b & 1 & (0.5) \\
\hline Invasión Capsular (\%) & 36 & $(17.0)$ \\
\hline No + invasión perineural (\%) & 104 & $(48.0)$ \\
\hline No PIN alto grado (\%) & 55 & (26.0) \\
\hline \multicolumn{3}{|l|}{ Estado ganglios linfáticos } \\
\hline $\begin{array}{l}\text { - Mediana ganglios } \\
\text { resecados por caso } \pm \text { DS } \\
\text { (rango) }\end{array}$ & \multicolumn{2}{|c|}{$3.6 \pm 3.3(0-16)$} \\
\hline - Ganglios positivos & & 0 \\
\hline
\end{tabular}

Evaluamos la función eréctil en pacientes que eran potentes preoperatoriamente (erecciones suficientes para la penetración vaginal) y que habían sido sometidos a preservación uni o bilateral (165). De estos, 93, tuvieron un seguimiento de 12 meses y 75 de ellos $(81 \%)$ eran potentes. El índice de potencia a las 12 semanas era del $54 \%$ y el $69 \%$ a los 6 meses. Se realizó un análisis de regresión de Cox para identificar los posibles factores predictivos para el retorno de la función sexual. Se encontraron los siguientes factores; edad del paciente $(p=0.005)$, preservación nerviosa $(p<0.001)$ y escala preoperatoria IIEF $(p=0.014)$. El IMC no tuvo impactó en los resultados de potencia.

Con respecto a la continencia urinaria, evaluamos un total de 324 pacientes intervenidos con dos técnicas diferentes, con (168) y sin (144) puboperineoplastia $(11,12)$. Las cifras de continencia a seis meses de toda la serie fueron del $78 \%$. Con la técnica antigua (sin puboperineoplastia), las cifras fueron $8.2 \%, 23 \%$, $58 \%$ y $92 \%$ a la semana, 6 semanas, 12 semanas y 1 año tras la cirugía respectivamente. Con la nueva modificación las cifras fueron de $26 \%, 59 \%$ y $80 \%$ a la semana, 6 semanas y 12 semanas respectivamente. Estas cifras fueron diferentes estadísticamente entre las dos técnicas ( $p<0.01$ ). (Tablas $\mathrm{VI}$ y VIII).

\section{CONCLUSIONES}

Hemos hecho un resumen de los aspectos más relevantes de nuestra técnica. Creemos que una de las contribuciones más importantes de la PRR se basará en su reproducibilidad.

La magnificación óptica y el avance de la tecnología deben hacer más fácil la enseñanza y aprendizaje de esta técnica. La meta real, control del cáncer, preservación de la continencia y de la potencia sexual representan un reto que se logra en poco más de $50 \%$ de los pacientes hoy en día, en manos de cirujanos experimentados. Esperamos que esta nueva técnica pueda mejorar estos resultados en el futuro. De ser así, se daría continuidad a lo que hoy representa un paradigma propuesto.

\section{NOTA DE LOS AUTORES}

Algunos nombres que se refieren a estructuras anatómica descritas en inglés, hemos preferido que figuraran sin traducción al español, para no añadir más confusión a la nomenclatura de la anatomía pélvica y prostática. 
TABLA III. MÁRGENES QUIRÚRGICOS (MQ) (2 15 PACIENTES).

\begin{tabular}{|c|c|c|}
\hline & (-)MQ & $T+1 M Q$ \\
\hline Porcentaje Global (\%) & $201(93.5)$ & $14(6.5)$ \\
\hline \multicolumn{3}{|c|}{ No estadio clínico (cTNM) (\%) } \\
\hline$\bullet \mathrm{Tlc}$ & $149(74.9)$ & $11(78.6)$ \\
\hline$\bullet \mathrm{T} 2$ & $48(24.1)$ & $3(21.4)$ \\
\hline$\bullet$ T3 & $2(1.0)$ & - \\
\hline \multicolumn{3}{|l|}{ No Gleason pieza (\%) } \\
\hline - 5 & $2(1.0)$ & - \\
\hline - 6 & $94(47.2)$ & $4(28.6)$ \\
\hline - $3+4$ & $83(41.8)$ & $5(35.7)$ \\
\hline - $4+3$ & 10 (5.0) & $3(21.4)$ \\
\hline$\bullet \geq 8$ & $10 \quad(5.0)$ & $2(14.3)$ \\
\hline \multicolumn{3}{|c|}{ No preop PSA (ng/ml) (\%) } \\
\hline$\bullet<2.5$ & $21(10.4)$ & $1 \quad(7.1)$ \\
\hline - $2.6-4.0$ & $41(20.4)$ & $2(14.3)$ \\
\hline - $4.1-10.0$ & $123(61.2)$ & $11(78.6)$ \\
\hline - $10.1-20.0$ & $12(6.0)$ & - \\
\hline$\cdot>20$ & $4 \quad(2.0)$ & . \\
\hline \multicolumn{3}{|c|}{ No estadio patológico (\%) } \\
\hline • PTO & $1 \quad(0.5)$ & - \\
\hline - PT2a & $37 \quad(18.4)$ & . \\
\hline - PT2b & $6 \quad(3.0)$ & $1(7.1)$ \\
\hline - $\mathrm{PT} 2 \mathrm{c}$ & $136(67.6)$ & $8(57.2)$ \\
\hline - PT3a & $13(6.5)$ & $3(21.5)$ \\
\hline - PT3b & $7(3.5)$ & $1(7.1)$ \\
\hline - PT4 & $1(0.5)$ & $1(7.1)$ \\
\hline
\end{tabular}

TABLA N. LOCALIZACIÓN DE MQ+ *

\begin{tabular}{|l|r|}
\hline Total de localizaciones & $16(14 \mathrm{pac})$ \\
Apical & $4(25)$ \\
Anterior & $2(13)$ \\
Cuello Vesical & $1(6)$ \\
Periférico & $1(6)$ \\
Lateral & $1(6)$ \\
Postero-Lateral & $3(19)$ \\
Posterior & $4(25)$ \\
\hline
\end{tabular}

*2 pacientes presentaron más de una localización 
TABLA V. RECURRENCIA PSA TRAS PRR (208 PACIENTES)*

\begin{tabular}{|l|r|r|}
\hline Mediana seguimiento PSA \pm DS (rango) & $9 \pm 5(1-21)$ & \\
\hline Recurrencia Global & No Pac (\%) & No recurrencia PSA (>0.2) (\%) \\
No estadio patológico (\%) & $194(93)$ & $14(7)$ \\
$\bullet$ pT2a & $37(19.7)$ & $2(28.6)$ \\
$\bullet$ pT2b & $7(3.7)$ & - \\
$\bullet$ pT2c & $144(76.6)$ & $5(71.4)$ \\
\hline
\end{tabular}

* Siete pacientes perdidos durante el seguimiento.

TABLA VI. POTENCIA SEXUAL: RESULTADOS.

\begin{tabular}{|c|c|c|}
\hline VARIABLES & MEDIA (MEDIANA) & RANGO \\
\hline \multicolumn{3}{|l|}{ PARAMETROS BASALES } \\
\hline EDAD & $59.62(58.64)$ & $43.16-74.14$ \\
\hline IMC & $27.94(27)$ & $19-50$ \\
\hline IPSS & $7.71(5)$ & $0-35$ \\
\hline (PSA) $\mathrm{ng} / \mathrm{ml}$ & $5.9(4.8)$ & $0.3-66.4$ \\
\hline \multicolumn{3}{|l|}{ POTENCIA SEXUAL } \\
\hline Potencia a las 12 semanas & \multicolumn{2}{|c|}{$54 \%$} \\
\hline Potencia a los 6 meses & \multicolumn{2}{|c|}{$69 \%$} \\
\hline Potencia a los 12 meses & \multicolumn{2}{|c|}{$81 \%$} \\
\hline
\end{tabular}

TABLA VII. CONTINENCIA URINARIA: RESULTADOS.

\begin{tabular}{|l|c|c|c|}
\hline Tiempo tras retirada de sonda vesical & $\begin{array}{c}\text { Técnica Antigua } \\
\mathbf{n = 1 4 4}\end{array}$ & $\begin{array}{c}\text { Técnica Nueva } \\
\mathbf{n = 1 6 8}\end{array}$ & P valor \\
\hline 1 semanas & $8.2 \%$ & $26 \%$ & $<0.01$ \\
6 semanas & $23 \%$ & $59 \%$ & $<0.01$ \\
12 semanas & $58 \%$ & $80 \%$ & $<0.01$ \\
24 semanas & $70 \%$ & $97 \%$ & $<0.01$ \\
1 año & $92 \%$ & SD & SD \\
\hline
\end{tabular}




\section{BIBLIOGRAFÍA y LECTURAS RECOMENDADAS (*lectura de interés $y^{* *}$ lectura fundamental)}

1. CATALONA, W.J.: "Potency, continence and complication rates in 1,870 consecutive radical retropubic prostatectomies". J. Urol., 162: 433, 1999.

2. GUILLONNEAU, B. y cols.: "Laparoscopic radical prostatectomy. Preliminary evaluation after 28 interventions". Presse Med., 27: 1570, 1998.

3. GUILLONNEAU, B. y cols.: "Laparoscopic radical prostatectomy: technical and early oncological assessment of 40 operations". Eur. Urol., 36: 14, 1999.

4. GUILLONNEAU, B. y cols.: "Update of the morbidity of radical retropubic prostatectomy: retrospective analysis of 100 consecutive operations during the period 1996-1997". Prog. Urol., 9: 662, 1999.

**5. MENON, M. y cols.: "Vattikuti institute prostatectomy: contemporary technique and analysis of results". Eur. Urol., 51: 648, 2007.

6. WALSH, P.C.; DONKER, P.J.: "Impotence following radical prostatectomy: insight into etiology and prevention". J. Urol., 128: 492, 1982.

*7. MENON, M.; TEWARI, A.; PEABODY, J.: "Vattikuti Institute prostatectomy: technique". J. Urol., 169: 2289, 2003.
*8. TEWARI, A. y cols.: "An operative and anatomic study to help in nerve sparing during laparoscopic and robotic radical prostatectomy". Eur. Urol., 43: 444, 2003.

**9. TEWARI, A. y cols.: "The proximal neurovascular plate and the tri-zonal neural architecture around the prostate gland: importance in the athermal robotic technique of nerve-sparing prostatectomy". BJU Int., 98: 314, 2006.

*10. TEWARI, A.K.; RAO, S.R.: “Anatomical foundations and surgical manoeuvres for precise identification of the prostatovesical junction during robotic radical prostatectomy". BJU Int., 984: 833, 2006.

**11. TAKENAKA, A. y cols.: "Preservation of the Puboprostatic Collar and Puboperineoplasty for Early Recovery of Urinary Continence after Robotic Prostatectomy: Anatomic Basis and Preliminary Outcomes". Eur. Urol., 2006.

**12. TAKENAKA, A. y cols.: "Preservation of the puboprostatic collar and puboperineoplasty for early recovery of urinary continence after robotic prostatectomy: anatomic basis and preliminary outcomes". Eur. Urol., 51: 433, 2007.

*13. GUILLONNEAU, B.; VALLANCIEN, G.: "Laparoscopic radical prostatectomy: the Montsouris technique". J. Urol., 163: 1643, 2000.

14. TEWARI, A. y cols.: "Identification of the retrotrigonal layer as a key anatomical landmark during robotically assisted radical prostatectomy". BJU Int., 98: 829, 2006. 